\title{
Creativity and Autonomy in Swarm Intelligence Systems
}

\author{
Mohammad Majid al-Rifaie • John Mark \\ Bishop • Suzanne Caines
}

Received: date / Accepted: date

\begin{abstract}
This work introduces two swarm intelligence algorithms - one mimicking the behaviour of one species of ants (Leptothorax acervorum) foraging (a 'Stochastic Diffusion Search', SDS) and the other algorithm mimicking the behaviour of birds flocking (a 'Particle Swarm Optimiser', PSO) - and outlines a novel integration strategy exploiting the local search properties of the PSO with global SDS behaviour. The resulting hybrid algorithm is used to sketch novel drawings of an input image, exploliting an artistic tension between the local behaviour of the 'birds flocking' - as they seek to follow the input sketch and the global behaviour of the 'ants foraging' - as they seek to encourage the flock to explore novel regions of the canvas. The paper concludes by exploring the putative 'creativity' of this hybrid swarm system in the philosophical light of the 'rhizome' and Deleuze's well known 'Orchid and Wasp' metaphor.
\end{abstract}

Keywords PSO · SDS · Autonomy · Swarm Intelligence • Visualisation • Computational Creativity

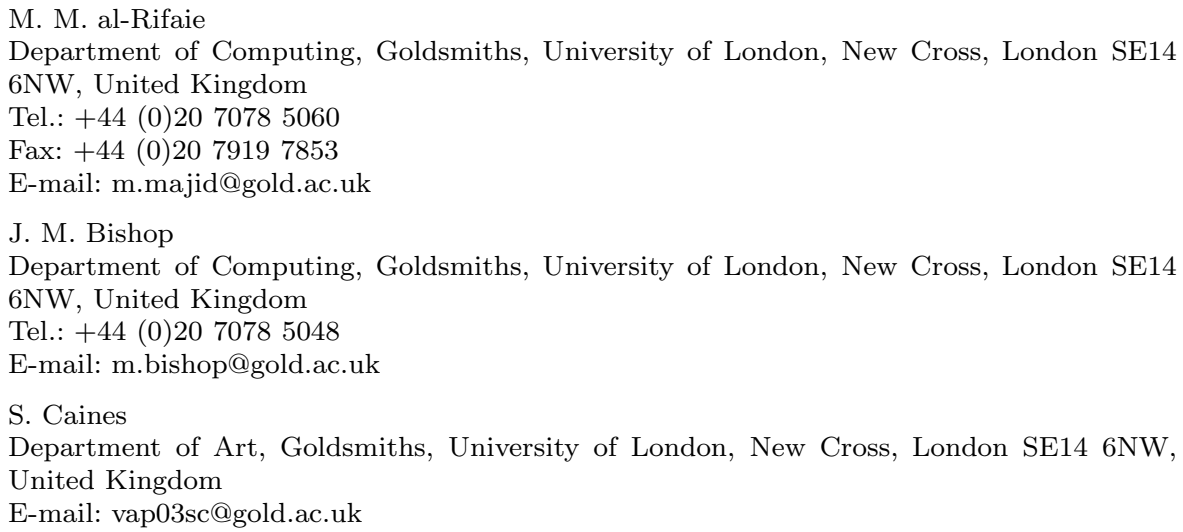




\section{Introduction}

In recent years, studies of the behaviour of social insects (e.g. ants and bees) and social animals (e.g. birds and fish) have proposed several new metaheuristics for use in collective intelligence. Natural examples of swarm intelligence that exhibit a form of social interaction are fish schooling, birds flocking, ant colonies in nesting and foraging, bacterial growth, animal herding, brood sorting etc.

This work explores creativity and autonomy through this type of collective intelligence, which emerges through the interaction of simple agents (representing the social insects/animals) in two nature-inspired algorithms, namely, Particle Swarm Optimisation (PSO) [21] and Stochastic Diffusion Search (SDS) [5].

Here, a user-made sketch is used as an input to the system. Then, the swarms of 'birds' and 'ants' explore the digital canvas they are provided with, going through the sketch and reworking it in their own way. The output of the system would be the swarms' 'interpretation' of the original sketch.

A-Life (Artificial Life), where the boundary between biology and artificial intelligence is blurred [23], inspired many artists and researchers in computer graphics to explore this area. Among the direct responses to A-Life are some works by Karl Sims (e.g. [44,45]). In an earlier work, Harold Cohen used techniques of artificial intelligence to produce art and developed a computer program called AARON, which produced drawings as well as paintings [25].

Following other works in the field of swarms painting $([30,2,48,49])$ and ant colony paintings $([17,29])$, the outputs discussed in this paper - created by the cooperation of birds and ants - are used as a platform to argue whether or not swarm intelligence algorithms have the potential to exhibit computationally creativity.

In this work, each of the swarm intelligence algorithms we use are explained, and an approach to their possible integration is highlighted. Subsequently, an explanation on how the new hybrid algorithm produces a drawing is presented. Lastly, while observing examples of human swarms, the similar individualistic approach of the computer generated nature-inspired swarms in making a drawing is highlighted, followed by a brief section on creativity in general as well as a discussion on whether swarms can be computationally creative. The creativity of swarms is also discussed in a non-representational form.

There are many works where the input of nature has been utilised, some of which are claimed be to art. In our discussion, we emphasise the importance of what we later define as 'Swarm Regulated Freedom' (SR freedom) cf. Gaussian Constrained Freedom (GC freedom) and the combinatorial creativity of the hybrid swarm system. Then we contrast it with examples of potential non-human assessment of aesthetic judgement and suggestions of creativity in natural distributed systems. Our modest conclusion would be that SR freedom (vs. GC freedom) as, for example, exhibited in the hybrid bird and ant algorithm presented herein, can be useful in generating interesting and intelligible 
drawing outputs. We conclude the paper with some suggestions for possible future research.

\section{Communication in Social Systems}

Communication - social interaction or information exchange - observed in social insects and social animals plays a significant role in all swarm intelligence algorithms, including SDS and PSOs. Although in nature it is not only the syntactical information that is exchanged between the individuals but also semantic rules and beliefs about how to process this information [22], in typical swarm intelligence algorithms only the syntactical exchange of information is taken into account.

In the study of the interaction of social insects, two important elements are the individuals and the environment, which result in two integration schemes: the first is the way in which individuals self-interact (interact with each other) and the second is the interaction of the individuals with the environment [8]. Self-interaction between individuals is carried out through recruitment strategies and it has been demonstrated that, typically, various recruitment strategies are used by ants [19] and honey bees. These recruitment strategies are used to attract other members of the society to gather around one or more desired areas, either for foraging purposes or for moving to a new nest site.

In general, there are many different forms of recruitment strategies used by social insects; these may take the form of global or local strategies; one-to-one or one-to-many communication; and the deployment stochastic or deterministic mechanisms. The nature of information sharing varies in different environments and with different types of social insects. Sometimes the information exchange is quite complex where, for example it might carry data about the direction, suitability of the target and the distance; or sometimes the information sharing is simply a stimulation forcing a certain triggered action. What all these recruitment and information exchange strategies have in common is distributing useful information throughout their community [27].

However, in many hive-based (flock-based) agents - like the ones deployed in this paper - the benefits of memory and communication seem obvious, but as argued in [42], these abilities are not beneficial in every environment, depending on the way resources are clustered throughout the environment and whether the quality of the food sources is sufficiently high.

The algorithms used in this paper both rely on memory and communication to enable the agents explore various parts of the search space; albeit the communication methods outlined herein are less greedy than the one presented in [42]. Furthermore, the particular effect communication has on the "creative" act of the swarm-based algorithms used in this work is under further investigation.

The parable of 'The Blind Men and the Elephant' suggests how social interactions can lead to more intelligent behaviour. This famous tale, set in verse by John Godfrey Saxe [41] in the 19th century, characterises six blind 
men approaching an elephant. They end up having six different ideas about the elephant, as each person has experienced only one aspect of the elephant's body: wall (elephant's side), spear (tusk), snake (trunk), tree (knee), fan (ear) and rope (tail). The moral of the story is to show how people build their beliefs by drawing them from incomplete information, derived from incomplete knowledge about the world [22]. If the blind men had been communicating about what they were experiencing, they would have possibly come up with the conclusion that they were exploring the heterogeneous qualities that make up an elephant.

\section{Birds: Particle Swarm Optimisation}

Particle Swarm Optimisation (PSO) is population based optimization technique developed in 1995 by Kennedy and Eberhart [21,14]. It came about as a result of an attempt to graphically simulate the choreography of fish schooling or birds flying (e.g. pigeons, starlings, and shorebirds) in coordinated flocks that show strong synchronisation in turning, initiation of flights and landing, despite the fact that experimental researches to find leaders in such flocks failed [18]. In particle swarms, although members of the swarm neither have knowledge about the global behaviour of the swarm nor a global information about the environment, the local interactions of the swarms result in complex collective behaviour, such as flocking, herding, schooling, exploration and foraging behaviour $[38,24,4,20]$.

\subsection{Standard/Basic PSO}

A swarm in PSO algorithm comprises of a number of particles and each particle represents a point in a multi-dimensional problem space. Particles in the swarm explore the problem space searching for the optimal position, which is defined by a fitness function. The position of each particle, $x$, is thus dependent on the particle's own experience and those of its neighbours. Each particle has a memory, containing the best position found so far during the course of the optimisation, which is called personal best (pbest or $p$ ). Whereas the best position so far found throughout the population, or the local neighbourhood, is called global best $\left(p_{g}\right)$ and local best $\left(p_{l}\right)$ respectively.

The standard PSO algorithm defines the position of each particle by adding a velocity to the current position. Here is the equation for updating the velocity of each particle:

$$
\begin{gathered}
v_{i d}^{t}=w v_{i d}^{t-1}+c_{1} r_{1}\left(p_{i d}-x_{i d}^{t-1}\right)+c_{2} r_{2}\left(p_{g d}-x_{i d}^{t-1}\right) \\
x_{i d}^{t}=v_{i d}^{t}+x_{i d}^{t-1}
\end{gathered}
$$

where $w$ is the inertia weight whose optimal value is problem dependent [43]; $\mathbf{v}_{i d}^{t-1}$ is the velocity vector of particle $i$ in dimension $d$ at time step $t-1 ; c_{1,2}$ 
are the learning factors (also referred to as acceleration constants) for personal best and neighbourhood best respectively (they are generally constant and are usually set to 2$) ; \mathbf{r}_{1,2}$ are random numbers adding stochasticity to the algorithm and they are drawn from a uniform distribution on the unit interval $U(0,1) ; \mathbf{p}_{i d}$ is the personal best position of particle $x_{i}$ in dimension $d$; and $p_{g d}$ is global best (or neighbourhood best).

Therefore, PSO optimisation is based on particles' individual experience and their social interaction with the particle swarms.

After updating the velocities of the particles, their new positions are determined. Algorithm 1 summarises the behaviour of PSO algorithm when dealing with a minimisation problem.

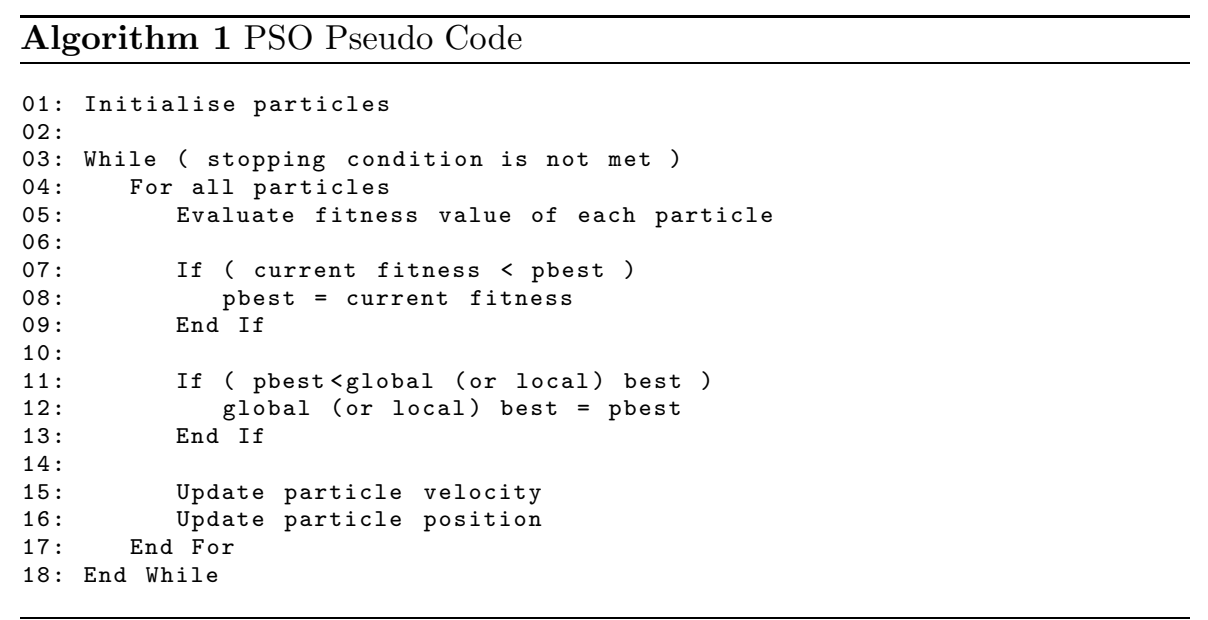

\section{Ants: Stochastic Diffusion Search}

This section introduces SDS [5], a multi-agent global search and optimisation algorithm, which is based on simple interaction of agents (inspired by one species of ants, Leptothorax acervorum, where a 'tandem calling' mechanism (one-to-one communication) is used, where the forager ant which finds the food location recruits a single ant upon its return to the nest and therefore the location of the food is physically publicised [28]). In SDS, direct one-to-one communication (which is similar to tandem calling recruitment) is utilised.

SDS introduced a new probabilistic approach for solving best-fit pattern recognition and matching problems. SDS, as a multi-agent population-based global search and optimisation algorithm, is a distributed mode of computation utilising interaction between simple agents [26].

Unlike many nature inspired search algorithms, SDS has a strong mathematical framework, which describes the behaviour of the algorithm by investigating its resource allocation [33], convergence to global optimum [34], 
robustness and minimal convergence criteria [31] and linear time complexity [35].

\subsection{SDS Architecture}

The SDS algorithm commences a search or optimisation by initialising its population. In any SDS search, each agent maintains a hypothesis, $h$, defining a possible problem solution. After initialisation two phases are followed (see Algorithm 2):

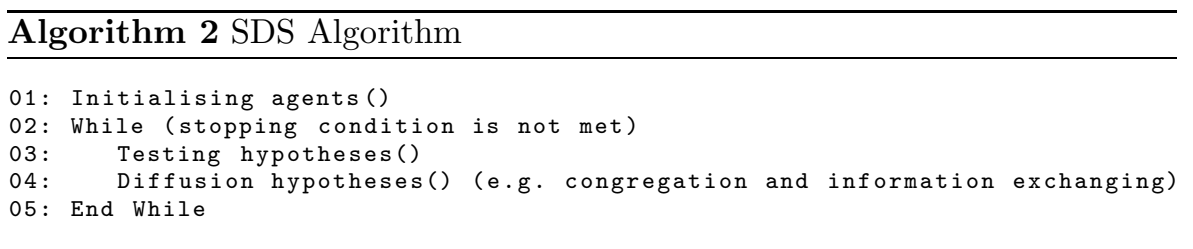

In the test phase, SDS checks whether the agent hypothesis is successful or not by performing a partial hypothesis evaluation which returns a boolean value (e.g. active or inactive). Later in the iteration, contingent on the precise recruitment strategy employed, successful hypotheses diffuse across the population and in this way information on potentially good solutions spreads throughout the entire population of agents.

In the Test phase, each agent performs partial function evaluation, $p F E$, which is some function of the agent's hypothesis; $p F E=f(h)$.

In the Diffusion phase, each agent recruits another agent for interaction and potential communication of hypothesis.

\subsection{Standard SDS and Passive Recruitment}

In standard SDS (as used in this paper), passive recruitment mode is employed. In this mode, if the agent is inactive, a second agent is randomly selected for diffusion; if the second agent is active, its hypothesis is communicated (diffused) to the inactive one. Otherwise there is no flow of information between agents; instead, a completely new hypothesis is generated for the first inactive agent at random (see Algorithm 3).

\section{Cooperation: Birds and Ants}

In an ongoing research, an initial set of experiments aimed to investigate if the information diffusion mechanism deployed in SDS ("ants") on its own improves PSO ("birds") behaviour; early results strongly indicate this is so (for detailed information and statistical analysis, see [1]). 


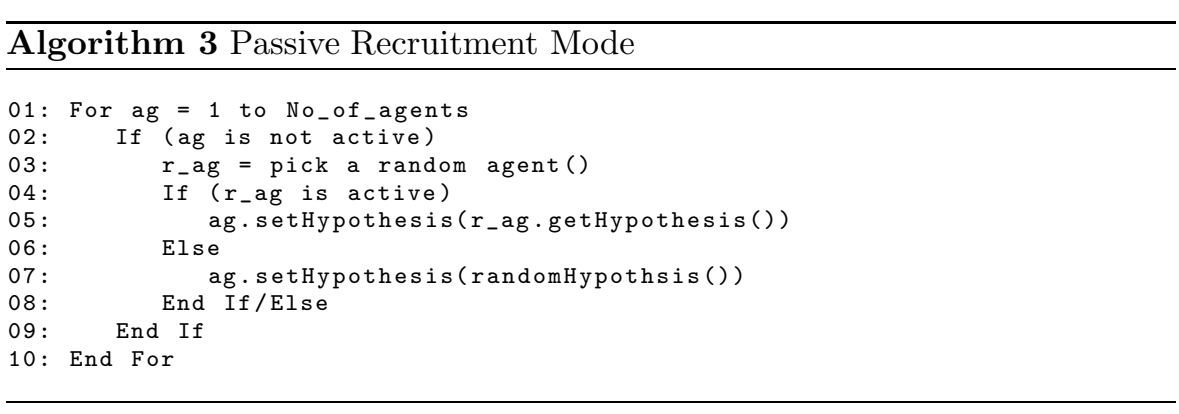

In the hybrid algorithm, each PSO particle has a current position, a memory (personal best position) and a velocity; each SDS agent, has a hypothesis and status (active or inactive).

In the experiment reported here, every particle in PSO is an SDS agent too - together termed pAgents. In pAgent, SDS hypotheses are defined by the PSO particle positions and an additional boolean variable (status) which determines whether the pAgent is active or inactive (see Figure 1).

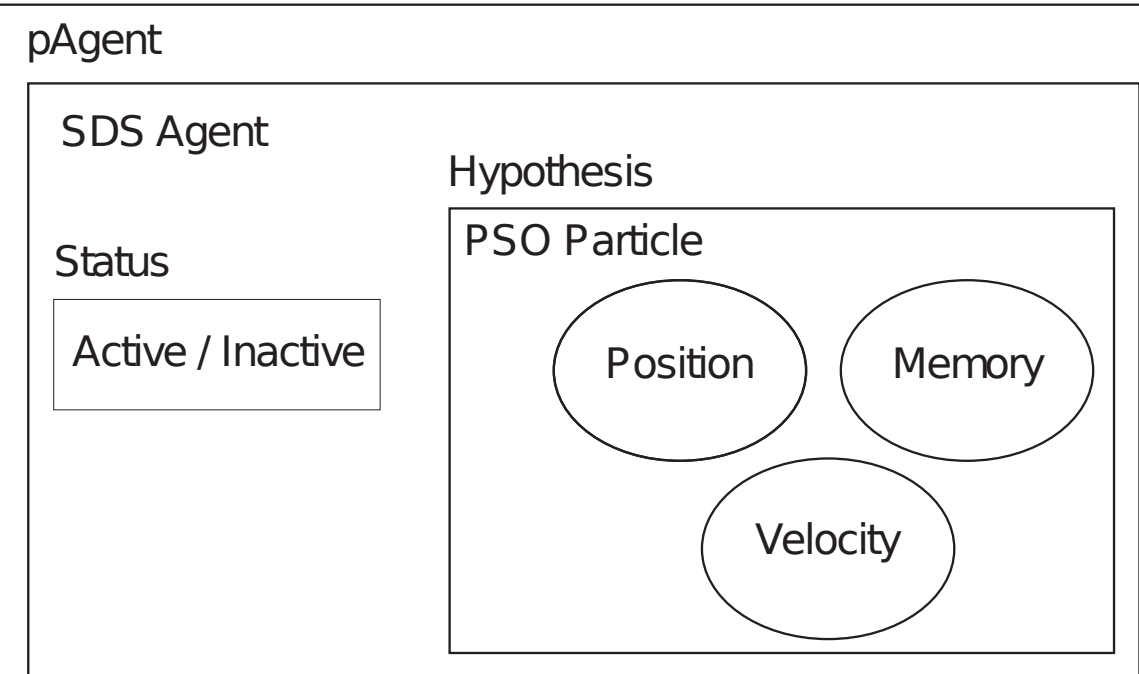

Fig. 1 pAgent. This figure shows the structure of pAgent.

The behaviour of the hybrid algorithm in its simplest form is presented in Algorithm 4.

In Algorithm 4, when the pAgents are initialised, pbests are initially set to the position of the particles and gbest is set to one of the particles randomly. In this work, the probability of SDS updating the position of one pAgent (i.e. pAg1) to the number of PSO function evaluations is 0.01 (see Lines 8). SDS is 
responsible for information sharing (Lines 26-27) and information dispensation (Lines 28-29).

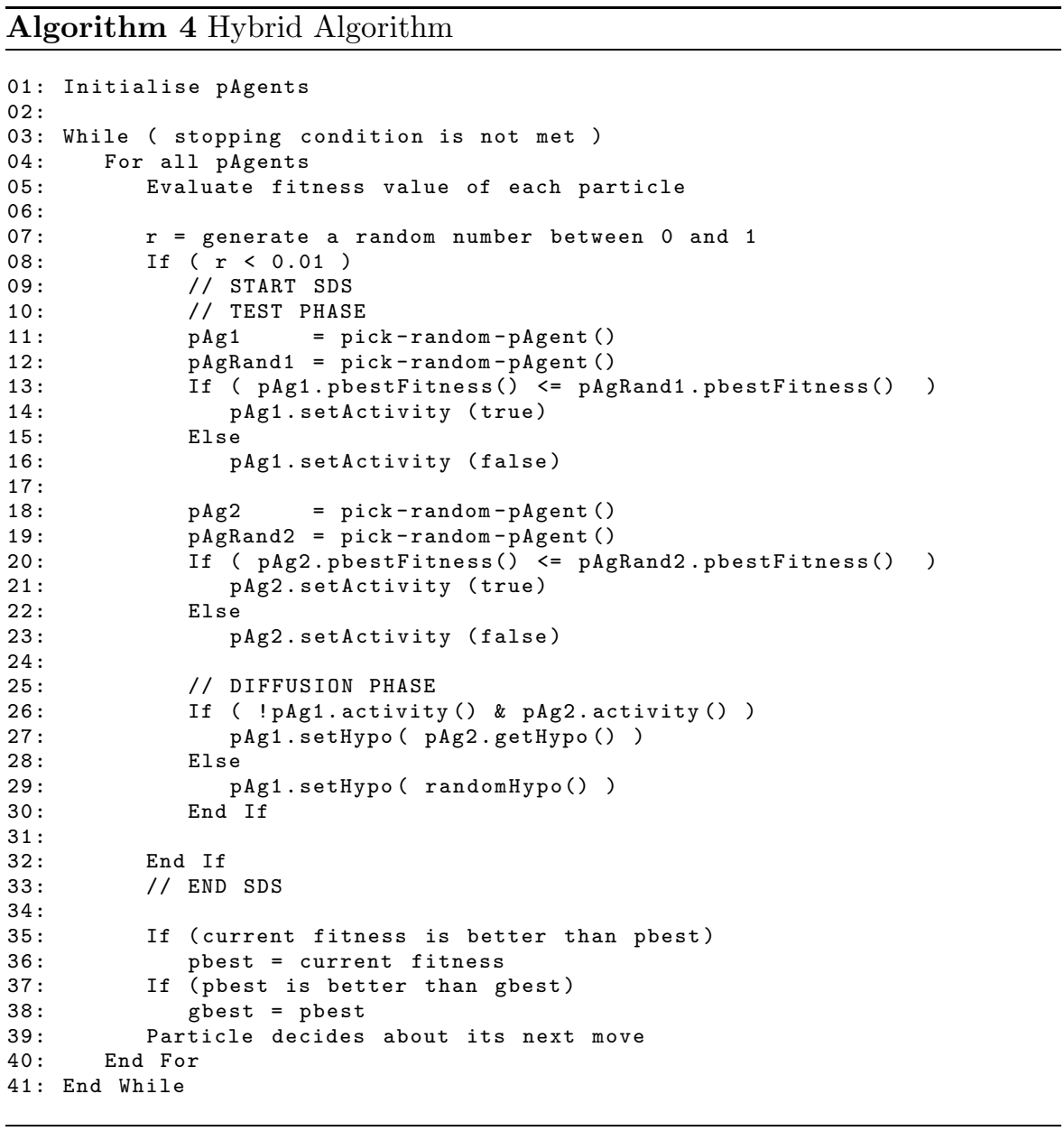

\section{The Drawing Mechanism}

This section explains how a sketch is provided to the hybrid swarm algorithm (PSO-SDS) and how the hybrid swarms make a drawing ${ }^{1}$ based on the original sketch.

\footnotetext{
1 For this work we consider a 'drawing' to be a representation of a target image, built up from an arrangement of lines which define its form; For the purposes of this work a drawing where all aspects of the original image are obscured is considered a poor 'drawing' of the target (albeit it may [or may-not] be an aesthetically pleasing object in its own right); a 'creative' drawing of the target is a drawing which differs noticeably from the original, whilst
} 


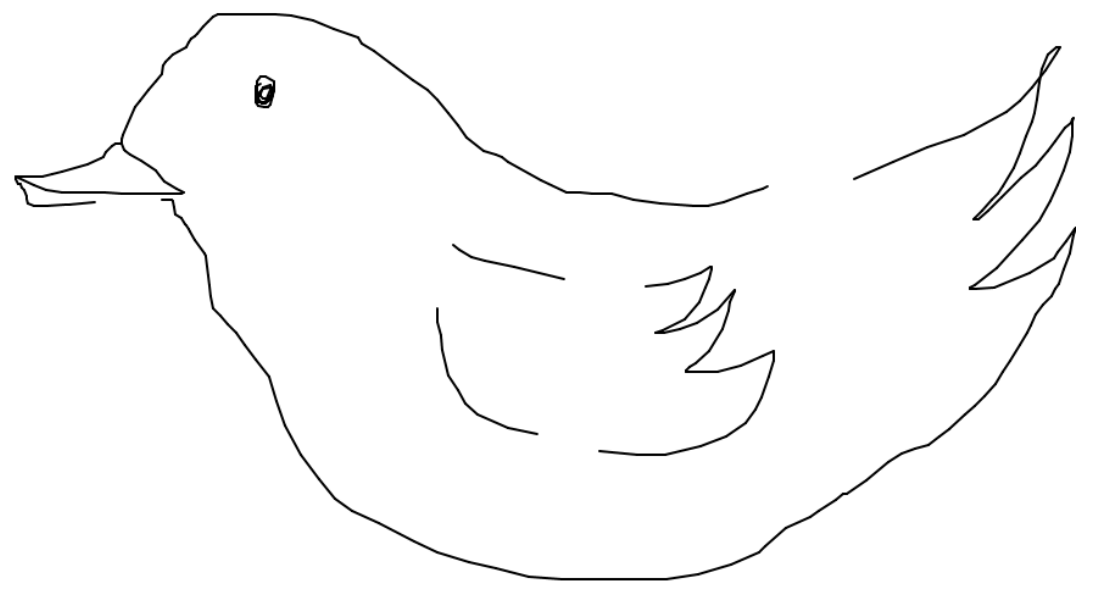

Fig. 2 Sketch: The sketch whose constituting points are used as input to the hybrid swarms.

\subsection{Birds and Ants Set off to Draw}

In the experimental setup of this work, a sketch is made on a screen with a mouse. Once the hybrid swarm (of 'birds' and 'ants') are presented with this sketch, which is a vector of $(x, y)$ coordinates corresponding to the points constituting the sketch (see Figure 2), they use it as an 'inspiration' and start making a drawing, which is based on the sketch, but utilises the swarms' 'style'.

Each one of the points is traced by the swarm as described in Algorithm 4. When the mouse pointer moves on the digital canvas to make a sketch, it is equivalent to moving the target. Each member of the swarm has the shape of a disc (with the centre representing the position of the particle) and as they move, their former position is connected to the current one with an arrow. In this way it can be said that 'the trace of the birds' / 'the footprint of the ants' stay on the canvas, creating a drawing inspired by the initial sketch (see Figure 3).

Therefore, the search space of the swarm is the canvas (a two dimensional array corresponding to the width and height of the canvas in pixels), where agents are initialised, and the goal of their performance is to 'trace' the points constituting the sketch. The swarms search on the canvas is terminated when

maintaining good correspondence [hi-fidelity] with at least some aspects of the original, such that the target image is still 'recognisable' in the resultant drawing. 


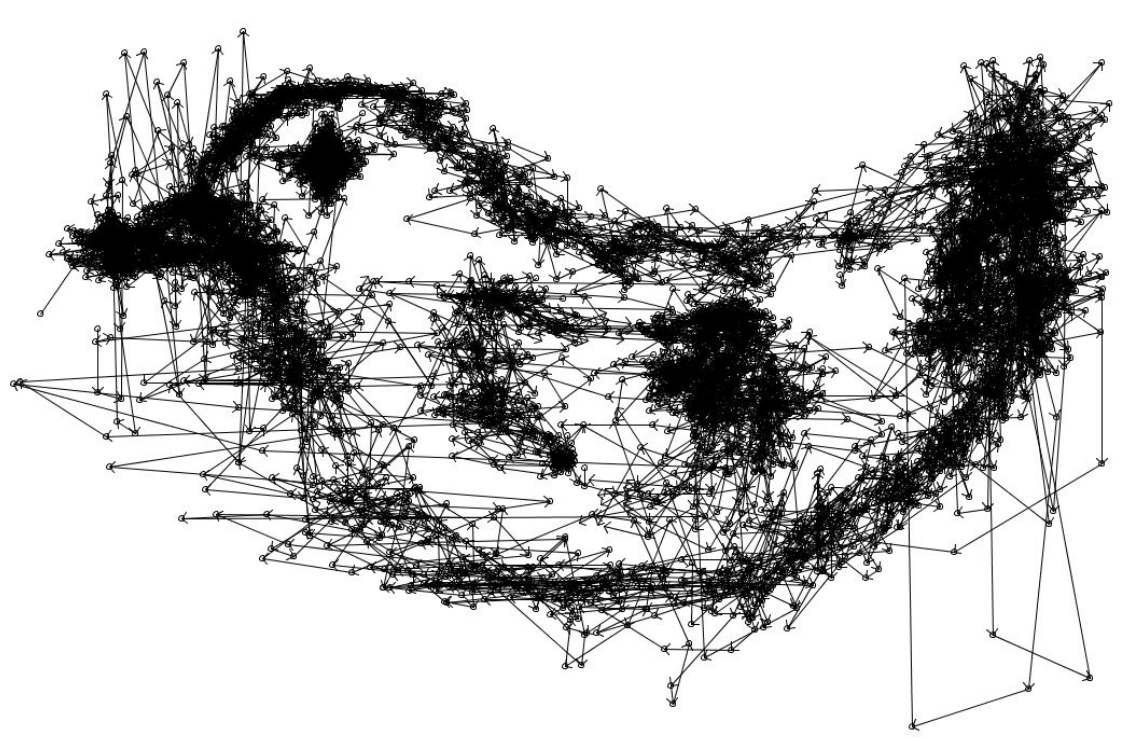

Fig. 3 The Drawing of the Hybrid Swarms (PSO + SDS).

they reach the end of the sketch (in other words, when there are no more points to consider).

In this context, gbest is the closest (fittest) particle to the point (on the sketch) being considered at any time. The hypotheses are the positions of each disc. The method used to determine whether an agent is active or inactive, and whether there should be information exchange, can be found in the test phase and diffusion phase of Algorithm 4 respectively. Twenty of the hybrid 'pAgents' were used in all the drawings of this work.

The next section presents a brief discussion on creativity, followed by a summary on whether swarms can show creativity in the 'artwork' they produce.

\section{Discussion on Creativity}

The goal of this section is to discuss whether the hybrid swarm algorithms have the potential to exhibit 'computational creativity' in what they draw and/or in the way they interact. 
7.1 On Freedom/Autonomy and Art

For many years, there has been discussion of the relationship between art, creativity and freedom; a debate elegantly encapsulated in the famous German prose by Ludwig Hevesi at the entrance of the Secession Building in Vienna:

"Der Zeit ihre Kunst

Der Kunst ihre Freiheit"

That is: "To Time its Art; To Art its Freedom".

Which, centuries after, resonates an earlier observation from Aristotle (384322 BCE) [15] emphasising the importance of freedom and autonomy (here, having "a tincture of madness") in presenting a creative act.

"There was never a genius without a tincture of madness."

On the other hand Margaret Boden, in [7], more recently argues that creativity has an ambiguous relationship with freedom:

"A style is a (culturally favoured) space of structural possibilities: not a painting, but a way of painting. Or a way of sculpting, or of composing fugues .. [] .. It's partly because of these [thinking] styles that creativity has an ambiguous relationship with freedom."

Among several definitions that have been given to creativity, around sixty of which (as stated by Taylor [47]) explore 'combinational creativity', which is defined as "the generation of unfamiliar combinations of familiar ideas" [6], a category we suggest that the presented outputs best fit in.

Considering the many factors constituting the evaluation of what is deemed 'creative', raises core issues regarding how humans evaluate creativity; their aesthetic capacity and potentially that of other animals (e.g. as exhibited in, say, mate-selection). Galanter [16] suggests that perhaps the 'computational equivalent' of a bird or an insect (e.g. in evaluating mate selection) is all that is required for [computational] aesthetic evaluation:

"This provides some hope for those who would follow a psychological path to computational aesthetic evaluation, because creatures with simpler brains than man practice mate selection."

In this context, as suggested in [13], the tastes of the individual in male bowerbirds are made visible when they gather collections of bones, glass, pebbles, shells, fruit, plastic and metal scraps from their environment, and arrange them to attract females [9]:

"They perform a mating dance within a specially prepared display court. The characteristics of an individual's dance or artefact display are specific to the species, but also to the capabilities and, apparently, the tastes of the individual." 
However the question of whether 'mate selection behaviour in animals implies making a judgement analogous to aesthetic judgement in humans' is perhaps (pace Nagel's famous discussion 'What is it like to be a bat? '[32]) a fundamentally unanswerable question.

In contrast, the role of education (or training) in recognising 'good' and 'bad', 'creative' and 'non-creative' has been experimentally probed. A suggestive study investigating this topic by Watanabe [50] gathers a set of children's paintings, and then adult humans are asked to label the "good" from the "bad". Pigeons are then trained through operant conditioning to only peck at good paintings. After the training, when pigeons are exposed to a novel set of already judged children's paintings, they show their ability in the correct classification of the paintings.

This emphasises the role of learning training and raises the question on whether humans are fundamentally trained (or "biased") to distinguish good and/or creative work.

Another tightly related topic to swarm intelligence in this context is the creativity of social systems. Bown in [10] indicates that our creative capabilities are contingent on the objects and infrastructure available to us, which help us achieve individual goals, in two ways:

"One way to look at this is, as Clark does [11], in terms of the mind being extended to a distributed system with an embodied brain at the centre, and surrounded by various other tools, from digits to digital computers. Another way is to step away from the centrality of human brains altogether and consider social complexes as distributed systems involving more or less cognitive elements."

Discussion on creativity and the conditions which make a particular work creative, have generated heated debate amongst scientists and philosophers for many years [40]; for a theoretical review on 'conditions of creativity'; the 'systems' view of creativity; cognitive approaches, etc see also [46]. Although this article does not aim to resolve any of these issues (or even suggest that the presented work strongly fits and endorses the category of the 'computationally creative realm'), we present an investigation of the performance of a novel swarm-intelligence drawing-system which, we suggest, highlights core issues inherent in exploring conceptual/artistic space(s). In turn this work is viewed through the philosophical lens of Deleuze, offering new insight on the putative creativity, autonomy and authorship of the resulting hybrid-swarm drawingsystem.

\section{On the "Creativity" of the Swarms}

As stated in the introduction, there have been several relevant attempts to create creative computer generated artwork using Artificial Intelligence, Artificial Life and Swarm Intelligence. Irrespective of whether the swarms are considered genuinely creative or not, their similar individualistic approach is not totally dissimilar to those of the "elephant artists" [51]: 
"After I have handed the loaded paintbrush to [the elephants], they proceed to paint in their own distinctive style, with delicate strokes or broad ones, gently dabbing the bristles on the paper or with a sweeping flourish, vertical lines or arcs and loops, ponderously or rapidly and so on. No two artists have the same style."

Similarly, as is discussed in the next section, if the same sketch is repeatedly given to the hybrid swarm architecture, the output drawings, made by the swarms, are never the same. In other words, even if the hybrid swarm mechanism (of birds and ants) process the same input several times, it will not make two identical drawings; furthermore, the outputs it produces are not merely randomised variants of the input. This can be demonstrated qualitatively by comparing the output of the hybrid swarm system with a simple randomised tracing algorithm, where each point in the sketch is surrounded with discs (similar to the pAgents) at a Gaussian random distance and direction (contrast Figures 4 and 5). The reason why the hybrid swarm drawings are different from the simple randomised sketch, is that the underlying PSO flocking component-algorithm constantly endeavours to accurately trace the input image whilst the SDS foraging component constantly endeavours to explore the wider canvas (i.e. together the two swarm mechanisms ensure high-level fidelity to the input without making an exact low-level copy of the original sketch) ${ }^{2}$.

Thus, despite the fact that the swarms are constrained by the rules they follow (rules that were defined earlier in the paper), the stochastic parts of the algorithms allow them to exhibit a swarm-controlled "regulated difference" rather than a simple "random difference".

\subsection{Swarm Regulated Autonomy versus Gaussian Constrained Autonomy}

The drawings in Figure 4 (top and middle) show two outputs from a simple randomised algorithm when configured to exhibit limited 'random' variations in behaviour (i.e. there is only small Gaussian random distance and direction from the points of the original sketch; we term such behaviour Gaussian Constrained (GC) freedom); comparing the two drawings, we note a lack of any significant difference between them. Furthermore, when more 'autonomy' is granted to the randomised algorithm (by increasing the variance in the underlying Gaussian, which allows the technique to explore broader areas of the canvas), the algorithm soon begins to deviate excessively from the original sketch. For this reason such randomisation results in a very poor - low fidelity - interpretation of the original sketch (Figure 4-bottom). In contrast, although the agents in the hybrid 'birds and ants swarms' are free to access any part

2 Although in principle, both functions (exploration and exploitation; local and global search of the conceptual space) could be carried out by either algorithm on its own, the basic SDS mechanism is not the best local optimiser and similarly a 'standard' PSO is not the best global optimiser, hence the motivation for exploring the properties of their hybridisation; promising early results of which have been reported elsewhere [1]. 

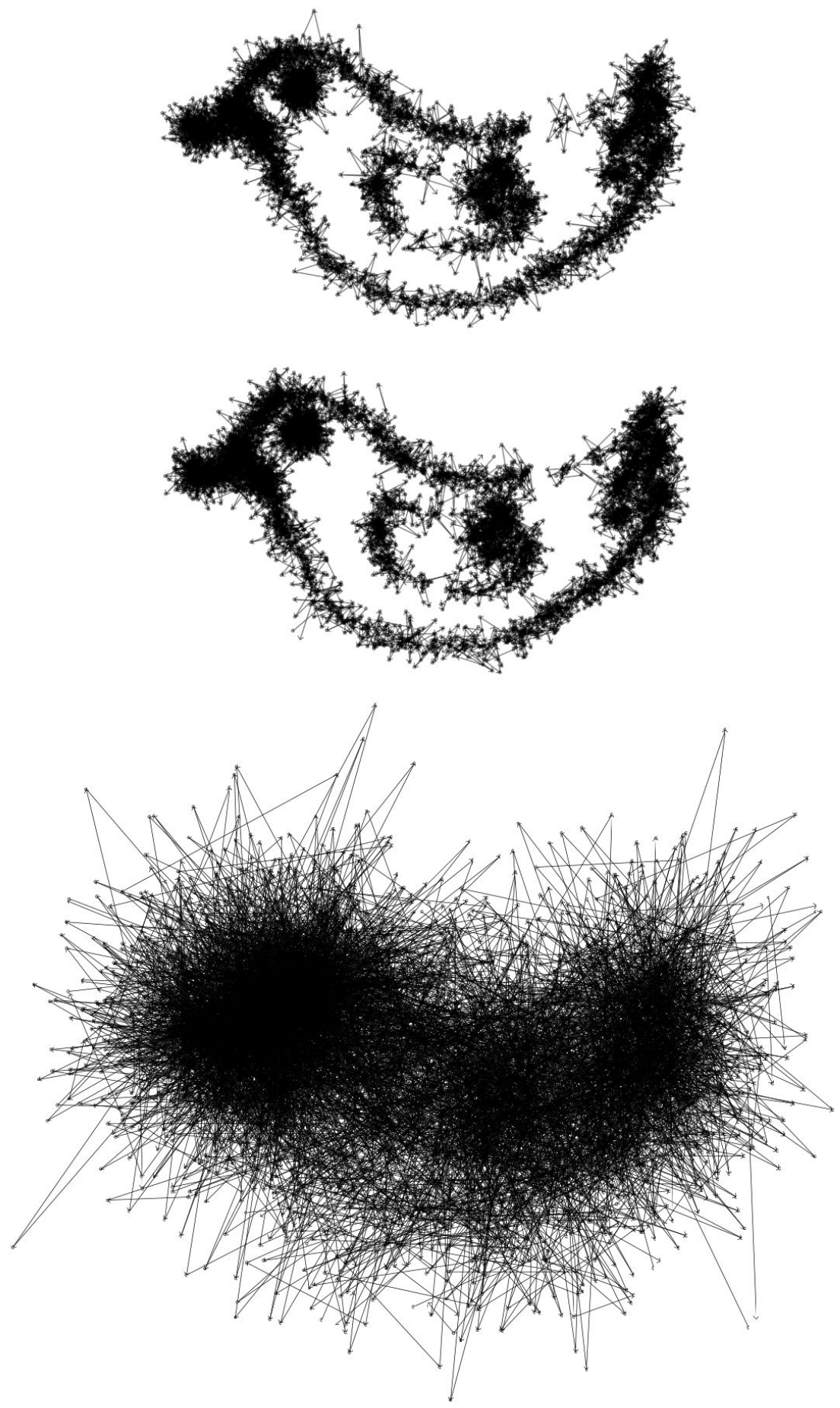

Fig. 4 The Drawings of the Swarms with Random Behaviour: This figure shows the drawings made with a simple randomised tracing algorithm, using Gaussian random distance and direction from the lines of the original sketch. The first two drawings (top and middle) use the same Gaussian random distance (e.g. $d$ ) and the bottom drawing uses the Gaussian random distance of $d \times 6$. 


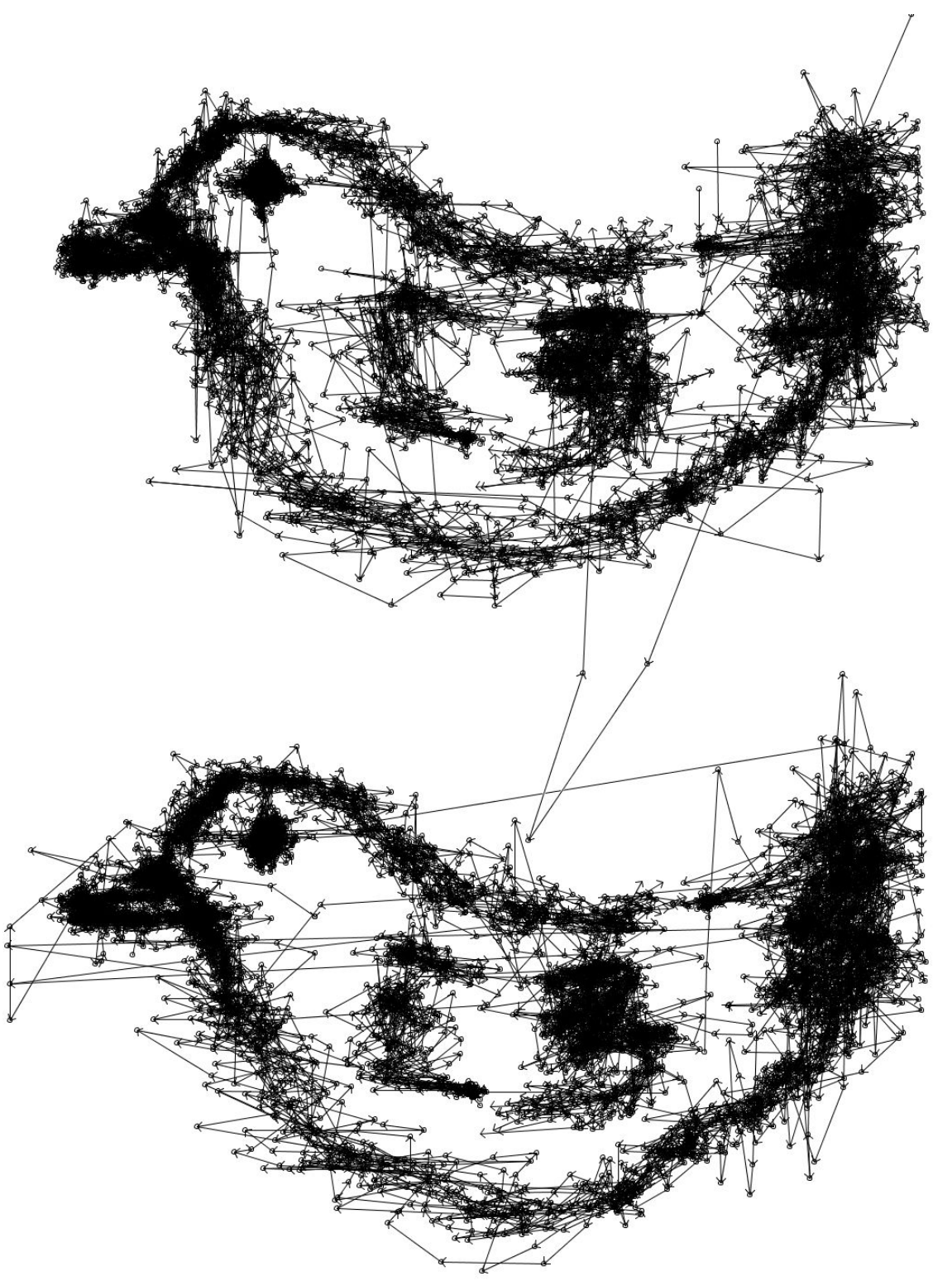

Fig. 5 Different Drawings of the Hybrid Swarms off a Single Sketch

of the canvas, the 'swarm-control' mechanism naturally enables the system to maintain recognisable fidelity to the original input. In this way, contra the hybrid system, it can be seen that simply by extending a basic swarm mechanism by giving it more randomised behaviour (giving it more 'random autonomy') fails to demonstrate that more 'creative drawings' would be produced. 
The Swarm Regulated freedom (SR freedom) or 'controlled freedom' (or the 'tincture of madness') exhibited by the hybrid swarm algorithm (induced by the stochastic side of the algorithms) is crucial to the resultant work and is the reason why having the same sketch does not result in the system producing identical drawings. This freedom emerges, among other things, from the stochasticity of SDS algorithm in picking agents for communication, as well as choosing agents to diffuse information; the tincture of madness in PSO algorithm is induced via its strategy of spreading the particles throughout the search space as well as the stochastic elements in deciding the next move of each particle. Although the algorithms (PSO and SDS) are natureinspired, we do not claim that the presented work is an accurate model of natural systems. Furthermore, whilst designing the algorithm there was no explicit 'Hundertwasser-like' attempt [37] by which we mean the stress on using curves instead of straight lines, as Hundertwasser considered straight lines not nature-like and tried not to use straight lines in his works to bias the style of the system's drawings.

\subsection{Human Swarm}

The autonomy or freedom that is explored in Swarm Regulated Freedom versus Gaussian Constrained Freedom could also be found in the human swarm currently being produced in contemporary art practices. Tino Sehgal - an artist employing swarm tactics into his art practice - has held several workshops in designated art galleries questioning whether it is "possible to maintain your place in the collective while asserting your individuality?" [39] and exploring the differences between the collective or swarm and the individual, and how the two can coexist - or not. In these workshops Sehgal gives participants simple instructions and guidelines in order to observe how human swarms function and appear visually from both a local and global perspective. Below, one of the participants (Dr Mary E Richards [39] describes Tino Sehgal's instructions for this workshop:

"One of the exercises we did on the second day was to walk together from one end of the [Tate Modern] Turbine Hall to the other (perhaps more as a collective than a swarm), with each length we were asked to slowly increase the speed with which we were travelling while at the same time maintaining our collectively. Other swarms were more free flowing and non-directional - starting together in a relatively close group, facing the same way and then moving with an awareness of each other but without anyone deliberately taking the lead."

Participants followed a natural leader, which created a global visual affect and local social interaction through visual perception (or one to one communication). Therefore, participants followed someone by looking at what direction they took and by keeping space between themselves and the other participants. This could be seen as similar interaction between participants and the 'tandem 
calling' mechanism (one-to-one communication) that we see in the forager ant, which finds the food location, recruits a single ant upon its return to the nest. Although limited, there appears to be some autonomy. Participants could walk slower and hang back from the group or they could overtake the leader.

The global visual affect that the human swarm takes could be judged as a visual representation effect. The participants have limited control over the decisions on the overall global appearance of the way the artwork looks, except for the local decisions they make on how and where to move; in a similar way the agents in the hybrid swarm system described earlier are able to move anywhere in the canvas, but they tend to follow the outline of the initial sketch. The global visuals that are created through the movements of the swarm (over which participants have some control, but restricted to the Turbine Hall space), could only be seen from an audience perspective in this instance.

\subsection{On Deleuze's Rhizome}

One of the philosophical frameworks which can help illuminate the claimed autonomy of the hybrid-swarm system is Deleuze's Rhizome. In this research, the concept of the rhizomatic 'assemblage' as exemplified by the wasp-orchid relation (as heterogeneous elements which form a rhizome) is explored and expanded to cover different aspects of the swarms performance. Deleuze et al. [12] in their 2004 book, A Thousand Plateaus, describe the concept as follows:

"How could movements of deterritorialization and processes of reterritorialization not be relative, always connected, caught up in one another? The orchid deterritorializes by forming an image, a tracing of a wasp; but the wasp reterritorializes on that image. The wasp is nevertheless deterritorialized, becoming a piece in the orchid as reproductive apparatus. But it reterritorializes the orchid by transporting its pollen. Wasp and orchid, as heterogeneous elements, form a rhizome. It could be said that the orchid imitates the wasp, reproducing its image in a signifying fashion (mimesis, mimicry, lure, etc.). But this is true only on the level of the strata - a parallelism between two strata such that a plant organization on one imitates an animal organization on the other. At the same time, something else entirely is going on: not imitation at all but a capture of code, surplus value of code, an increase in valence, a veritable becoming, a becoming-wasp of the orchid and a becoming-orchid of the wasp. Each of these becomings brings about the deterritorialization of one term and the reterritorialization of the other; the two becomings interlink and form relays in a circulation of intensities pushing the deterritorialization ever further. There is neither imitation nor resemblance, only an exploding of two heterogeneous series on the line of flight composed by a common rhizome that can no longer be attributed to or subjugated by anything signifying."

In the example of the wasp and the orchid, each are involved less in mimesis than in a network of becoming : the wasp "becomes" orchid, just as the orchid 
"becomes" wasp; they each form a rhizome with the other, an exchanging or capturing of each other's 'codes' or formal behavioural characteristics through the mutual formation of novel structural schemata for each entity.

In the context of this paper, and at each moment in time, when the movement of one PSO particle is considered (see section 3), the particle itself could be seen as adopting the role of a wasp and the cognitive and social components (particle's best position - memory - and global best position respectively) the role of the orchid, attracting the wasp to fly towards them (via communication). Since the moving particle might discover a better position (based on its fitness value) than its current cognitive/social components, the roles of wasp and orchid are interchangeable.

The same principle applies to the SDS mechanism; during the diffusion phase, when an inactive agent randomly chooses an active agent, the inactive agent takes the role of a wasp and the active agent take the role of an orchid, sharing its informational 'valence' with the wasp (inactive agent). Since an inactive agent can be active in subsequent iterations and the active agent could be inactive, the roles of wasp and orchid are interchangeable in SDS too.

Deleuze's wasp and orchid coupling in a Thousand Plateaus [12] challenges the concept of copy and the traditional notion that the copy is only as good as the nearness to its original; here 'mimicry' is already an activity of mutual transformation, emerging as neither good nor bad but rather as a constant experimentation in an intensive state of becoming and this is where creativity lies.

Another example of human swarm is shared research where there is a 'swarming of knowledge' around a topic. The topic is the focus and it controls the freedom of the swarm. The sharing in this case happens through examples of work, writing as well as other sources. This goes along the performance of the swarm intelligence algorithm that enacts the behaviour of one species of ants - Leptothorax acervorum - foraging (Stochastic Diffusion Search) and other algorithm that simulates the behaviour of birds flocking (Particle Swarm optimization). Thus, when one agent interacts with the other, they can influence or being influenced (using both their global best and memory); each agent can interchangeably enact the role of the orchid and the wasp in constant state of becoming as they trace the initial sketch on the canvas, using the rules through which autonomy expresses itself - the 'rules' that although enable the wasps to have the autonomy to fly anywhere on the canvas, still motivate them to be influenced by the orchids.

In this way, whenever the swarms produce a drawing using the initial sketch, the effects that the "wasps" create each time exhibits different states underlined by a process of becoming: which has a visual expression (through the trace of the members of the swarms on the canvas), and a non-visual expression (via information exchange between the members of the swarms). Observing the drawing process from a higher level of abstract activity and being inspired by Deleuze and Guattari's Rhizome, the initial sketch can be associated with the orchid, aiming to attract the wasps (swarms population) 
to itself. The final drawings of the swarms, despite giving the freedom and autonomy, still aim to stay loyal to the initial sketch.

In the swarm intelligence algorithms used in this work, the movement of agents and their communication are tightly coupled as the agents depend on the communication they have with each other in order to ensure movement. At each point in time, when the initial sketch is being traced by the swarms, each agent is deterritorialising as it utilises its personal and global bests to move, and then reterritorialises itself onto the next agent when they interact. The agents move through the use of the cognition and social components, reproducing an original drawing in a form of mimicry - which is not the same as other instances of the swarms' original drawings from a single sketch - thus creating another new image of itself in a constant state of becoming; this state of mimicry is not copying but a capture of formal characteristics.

As discussed earlier, communication between the agents is essential for the production of the final drawing; however the drawings do not register the communication (no visualisation showing which agent is communicating with which at each step in time). As Simon O'Sullivan [36] suggests, for example, creativity does not need to be evaluated in visual terms. Therefore, instead of judging the creativity of the swarms solely on the drawings they produce, other factors which might suggest creativity should be taken into account. Here, the constant state of becoming can be perceived as the non-visible layer of communication that happens between the members of the swarm.

Anthropologists also use the term 'deterritorialized' as a weakened set of ties to a place and that certain memories tend to transcend boundaries and movement of time, which indicates the change of culture that still relies on anchors [3]. At that moment the deterritorializing process begins when the local culture is enclosed by the global community. A deteritoralization takes place when the local culture becomes global. As communities move into different locations, they take on a different meaning that they previously held; they are reterritorialized culturally and become part of the global culture. Similar to the behaviour of swarm intelligence algorithms, the local interactions between agents lead to the global behaviour of the swarm. They form a symbiotic relationship between local and global.

\section{Conclusion}

In this paper, we have discussed the possibility for the exhibition of 'computational creativity' by a novel hybrid-swarm algorithm. This specific work described herein uses swarm intelligence techniques to explore the difference between using Gaussian Constrained Autonomy (GC autonomy) and Swarm Regulated Autonomy (SR autonomy) in the production of 'traced' line drawings; our work highlights the features of swarm-regulated difference versus simple-random difference (exploration) in the production of such 'drawings' by computer. 
The computational artist so described is the outcome of a novel marriage between two classical swarm intelligence algorithms (PSO and SDS) whose scientific value is currently being investigated [1]. We raise the question of whether integrating swarm intelligence algorithms (inspired by social systems in nature) could possibly lead to a different way of exploring creative spaces and whether the swarms demonstrate computational creativity in a representational and non-representational way.

\section{References}

1. al-Rifaie, M.M., Bishop, M., Blackwell, T.: An investigation into the merger of stochastic diffusion search and particle swarm optimisation. In: GECCO '11: Proceedings of the 2011 GECCO conference companion on Genetic and evolutionary computation, pp. 37-44. ACM, New York, NY, USA (2011)

2. Aupetit, S., Bordeau, V., Monmarche, N., Slimane, M., Venturini, G.: Interactive evolution of ant paintings. In: The 2003 Congress on Evolutionary Computation, 2003. CEC'03., vol. 2, pp. 1376-1383 (2004)

3. Basch, L.G.S.: Nations Unbound: Transnational Projects, Postcolonial Predicaments and Deterritorialized Nation-states, 1 edn. Routledge (1993)

4. Bayazit, O.B., Lien, J.M., Amato, N.M.: Roadmap-based flocking for complex environments. In: PG '02: Proceedings of the 10th Pacific Conference on Computer Graphics and Applications, p. 104. IEEE Computer Society, Washington, DC, USA (2002)

5. Bishop, J.: Stochastic searching networks. pp. 329-331. Proc. 1st IEE Conf. on Artificial Neural Networks, London, UK (1989)

6. Boden, M.: Creativity in a nutshell. Think 5(15), 83-96 (2007)

7. Boden, M.: Creativity and Art: Three Roads to Surprise. Oxford University Press (2010)

8. Bonabeau, E., Dorigo, M., Theraulaz, G.: Inspiration for optimization from social insect behaviour. Nature 406, 3942 (2000)

9. Borgia, G.: Complex male display and female choice in the spotted bowerbird: specialized functions for different bower decorations. Animal Behaviour 49, 1291-1301 (1995)

10. Bown, O.: Generative and adaptive creativity. In: J. McCormack, M. d'Inverno (eds.) In Computers and Creativity. Berlin: Springer (2011)

11. Clark, A.: Natural-born cyborgs: Minds, technologies, and the future of human intelligence. Oxford University Press (2003)

12. Deleuze, G., Guattari, F., Massumi, B.: A thousand plateaus. University of Minnesota Press Minneapolis (2004)

13. Dorin, A., Korb, K.: Creativity refined. in computers and creativity. In: J. McCormack, M. d'Inverno (eds.) In Computers and Creativity. Berlin: Springer (2011)

14. Eberhart, R., Kennedy, J.: A new optimizer using particle swarm theory. In: Proceedings of the sixth international symposium on micro machine and human science, vol. 43. New York, NY, USA: IEEE (1995)

15. Etzioni, A., Ben-Barak, A., Peron, S., Durandy, A.: Ataxia-telangiectasia in twins presenting as autosomal recessive hyper-immunoglobulin $\mathrm{m}$ syndrome. IMAJ-RAMAT GAN-9(5), 406 (2007)

16. Galanter, P.: Computational aesthetic evaluation: Past and future. In: J. McCormack, M. d'Inverno (eds.) In Computers and Creativity. Berlin: Springer (2011)

17. Greenfield, G.: Evolutionary methods for ant colony paintings. APPLICATIONS OF EVOLUTIONARY COMPUTING, PROCEEDINGS 3449, 478-487 (2005)

18. Heppner, F., Grenander, U.: A stochastic nonlinear model for coordinated bird flocks. American Association for the Advancement of Science, Washington, DC(USA). (1990)

19. Holldobler, B., Wilson, E.O.: The Ants. Springer-Verlag (1990)

20. Janson, C.H.: Experimental evidence for spatial memory in foraging wild capuchin monkeys,cebus apella. Animal Behaviour 55, 1229-1243 (1998)

21. Kennedy, J., Eberhart, R.C.: Particle swarm optimization. In: Proceedings of the IEEE International Conference on Neural Networks, vol. IV, pp. 1942-1948. IEEE Service Center, Piscataway, NJ (1995) 
22. Kennedy, J.F., Eberhart, R.C., Shi, Y.: Swarm intelligence. Morgan Kaufmann Publishers, San Francisco ; London (2001)

23. Levy, S.: Artificial life: A report from the frontier where computers meet biology. Vintage Books (1993)

24. Mataric, M.: Interaction and intelligent behavior. Ph.D. thesis, Department of Electrical, Electronics and Computer Engineering, MIT, USA (1994)

25. McCorduck, P.: Aaron's code: meta-art, artificial intelligence, and the work of Harold Cohen. WH Freeman (1991)

26. de Meyer, K., Bishop, J.M., Nasuto, S.J.: Stochastic diffusion: Using recruitment for search. Evolvability and interaction: evolutionary substrates of communication, signalling, and perception in the dynamics of social complexity (ed. P. McOwan, K. Dautenhahn \& CL Nehaniv) Technical Report 393, 60-65 (2003)

27. de Meyer, K., Nasuto, S., Bishop, J.: Stochastic diffusion optimisation: the application of partial function evaluation and stochastic recruitment in swarm intelligence optimisation. Springer Verlag 2, Chapter 12 in Abraham, A. and Grosam, C. and Ramos, V. (eds), "Swarm intelligence and data mining" (2006)

28. Moglich, M., Maschwitz, U., Holldobler, B.: Tandem calling: A new kind of signal in ant communication. Science 186(4168), 1046-1047 (1974)

29. Monmarche, N., Aupetit, S., Bordeau, V., Slimane, M., Venturini, G.: Interactive evolution of ant paintings. In: B.M.e. al (ed.) 2003 Congress on Evolutionary Computation, vol. 2, pp. 1376-1383. IEEE Press (2003)

30. Moura, L., Ramos, V.: Swarm paintings-nonhuman art. ARCHITOPIA book, art, architecture and science pp. 5-24 (2007)

31. Myatt, D.R., Bishop, J.M., Nasuto, S.J.: Minimum stable convergence criteria for stochastic diffusion search. Electronics Letters 40(2), 112-113 (2004)

32. Nagel, T.: What is it like to be a bat? The Philosophical Review 83(4), 435-450 (1974)

33. Nasuto, S.J.: Resource allocation analysis of the stochastic diffusion search. Ph.D. thesis, University of Reading, Reading, UK (1999)

34. Nasuto, S.J., Bishop, J.M.: Convergence analysis of stochastic diffusion search. Parallel Algorithms and Applications 14(2) (1999)

35. Nasuto, S.J., Bishop, J.M., Lauria, S.: Time complexity of stochastic diffusion search. Neural Computation NC98 (1998)

36. O'Sullivan, S., et al.: Art encounters Deleuze and Guattari: Thought beyond representation. Palgrave Macmillan Basingstoke (2006)

37. Restany, P.: Hundertwasser: the painter-king with the five skins: the power of art. Taschen America Llc (2001)

38. Reynolds, C.W.: Flocks, herds, and schools: A distributed behavioral model. Computer Graphics 21(4), 25-34 (1987)

39. Richards, M.E., Caines, S.: Unconventional encounters. Performance Research Journal (2012). Submitted

40. Rothenberg, A., Hausman, C.: The creativity question. Duke University Press Books (1976)

41. Saxe, J.G., Lathen, D., Chief, B.: The Blind Man and the Elephant. The Poems of John Godfrey Saxe (1882)

42. Schermerhorn, P., Scheutz, M.: The impact of communication and memory in hivebased foraging agents. In: Artificial Life, 2009. ALife'09. IEEE Symposium on, pp. 29-36 (2009)

43. Shi, Y., Eberhart, R.C.: Parameter selection in particle swarm optimization. Lecture notes in computer science pp. 591-600 (1998)

44. Sims, K.: Artificial evolution for computer graphics. Computer Graphics 25(4), 319-328 (1991)

45. Sims, K.: Evolving 3d morphology and behavior by competition. Artificial Life 1(4), 353-372 (1994)

46. Sternberg, R.: The nature of creativity: Contemporary psychological perspectives. Cambridge Univ Pr (1988)

47. Taylor, C.: 4 Various approaches to and definitions of creativity. The nature of creativity: Contemporary psychological perspectives p. 99 (1988)

48. Urbano, P.: Playing in the pheromone playground: Experiences in swarm painting. Applications on Evolutionary Computing pp. 527-532 (2005) 
49. Urbano, P.: Consensual paintings. Applications of Evolutionary Computing pp. 622-632 (2006)

50. Watanabe, S.: Pigeons can discriminate "good" and "bad" paintings by children. Animal Cognition 13(1) (2009)

51. Weesatchanam, A.M.: Are Paintings by Elephants Really Art? The Elephant Art Gallery (2006) 